\title{
THE STOCHASTISATION HYPOTHESIS AND THE SPACING OF PLANETARY SYSTEMS
}

\author{
by
}

Jacky Cresson ${ }^{1,2}$

${ }^{1}$ Laboratoire de Mathématiques Appliquées de Pau, Université de Pau et des Pays de l'Adour, avenue de l'Université, BP 1155, 64013 Pau Cedex, France

${ }^{2}$ Institut de Mécanique Céleste et de Calcul des Éphémérides, Observatoire de Paris,

77 avenue Denfert-Rochereau, 75014 Paris, France

Il était générallement admis que les probabilités n'étaient que l'expression de notre ignorance. Pour les systèmes instables, il n'en est pas ainsi. Les probabilités acquièrent une signification dynamique intrinsèque. Peu à peu se dessine dès lors une nouvelle rationalité dans laquelle probabilité n'est pas ignorance et science ne se confond pas avec certitude. C'est à ce prix que la notion d'évolution et avec elle les notions d'événement et de créativité font leur entrée dans les lois fondamentales de la nature.

Ilya Progogine, Les lois du Chaos, Ed. Flammarion, 1994

\begin{abstract}
We introduce the stochastisation hypothesis which aims to provide a framework to deal with physical systems in random environment. We apply the stochastisation hypothesis in two different cases : in the study of the dynamics of a protoplanetary nebula and in the Chaotic long term behaviour of a generic planetary system using previous works of S. Albeverio and al., L. Nottale and J. Laskar. These results give both a particular law for the distribution of planetary orbits.
\end{abstract}

\section{Contents}

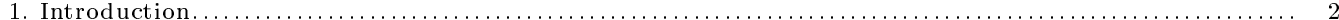

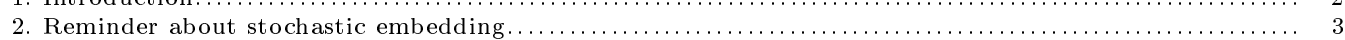

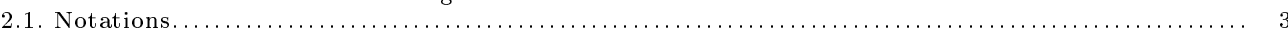

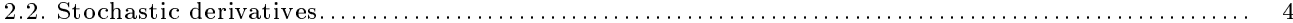

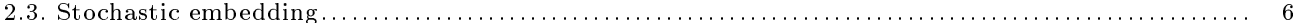

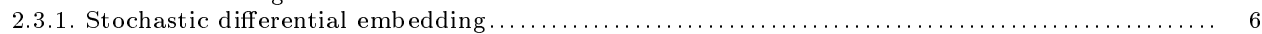

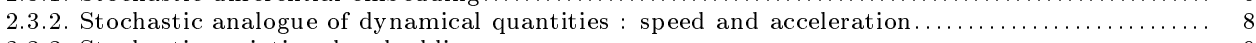

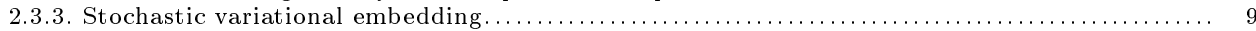

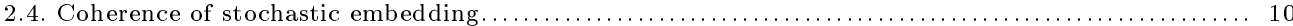

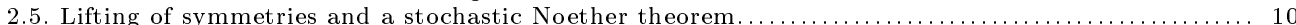

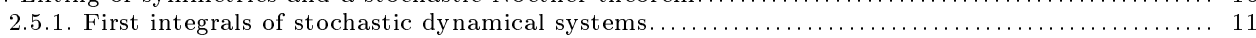

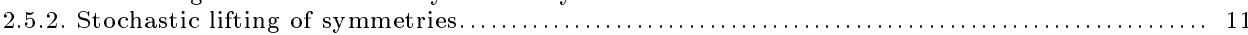

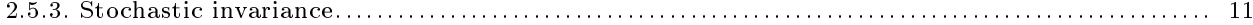

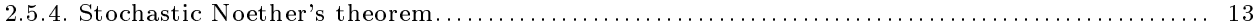

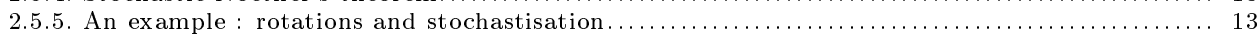

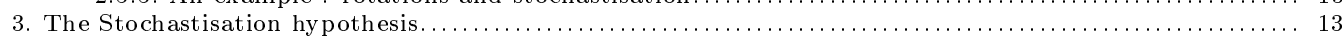

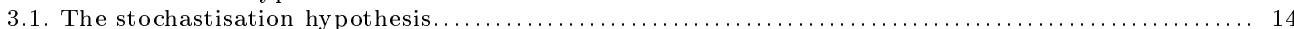

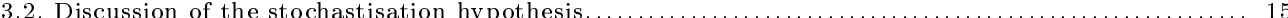

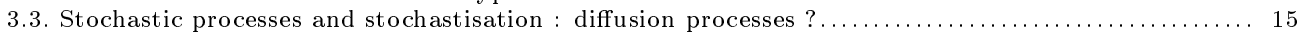




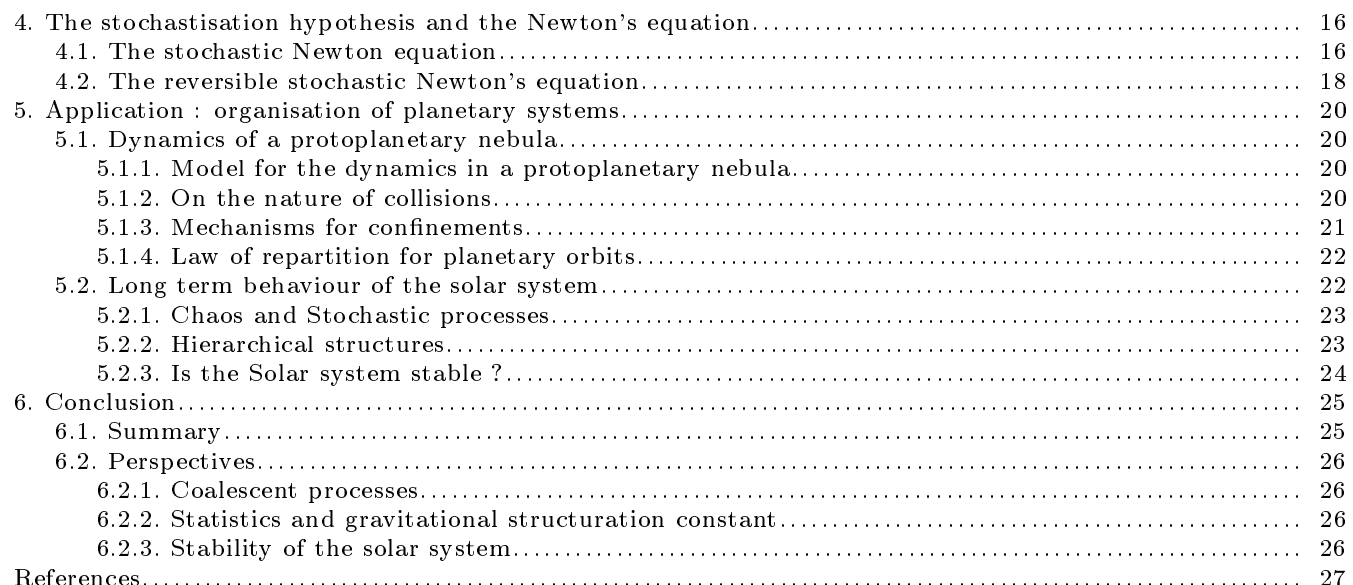

\section{Introduction}

The aim of this paper is to describe a new way to take into account the bath of a given physical experiment on the dynamical equations which model it. As a typical example, we think to the Davidov's model of energy transfer in proteins where there exists a phonon bath or the classical problem of the Brownian motion. From the mathematical point of view, our approach is based on the recent stochastic embedding formalism of Lagrangian systems developed by S. Darses and the author in [10].

Our main concern is the following stochastisation problem :

i - Let $x_{t}$ be a dynamical variable governed by a physical constraint. We assume in the following that the dynamics of $x_{t}$ is controlled by a Lagrangian equation, i.e. there exists a Lagrangian $L$ such that $x_{t}$ satisfies the classical Euler-Lagrange equations. From the physical point of view, it means that we look for Physical systems coming from a least action principle.

ii - We assume that the underlying bath, which is assumed to model the particular conditions under which the experiment is done, induces random behaviours, i.e. that $x_{t}$ is now no longer deterministic but a stochastic process $X_{t}$.

What is the dynamics of $X_{t}$ ?

In this paper, we will use the following notion of stochastisation, which is in fact an hypothesis of a physical nature on the underlying dynamics: 
Stochastization hypothesis : The dynamics of $X_{t}$ is obtain by the stochastic embedding in the sense of $[\mathbf{1 0}]$ of the initial Lagrangian system.

The previous framework can be used in a wild variety of problems. We have an analogous situation when one studies the long term behaviour of chaotic dynamical systems or the dynamics of a protoplanetary nebula. In this case we have no longer a bath but the long term dynamics can be described using stochastic processes $([\mathbf{2 8}],[\mathbf{2 9}])$. Using the stochastisation hypothesis, we prove that the law for the distribution of planetary orbits follows a simple rule. This work is related to L. Nottale's approach to chaotic dynamical systems [41] and its application to the Titius-Bode law [42].

The plane of our paper is as follow : In Section 2 we remind the stochastic embedding of dynamical systems developed in [10]. In Section 3 we define the stochastisation hypothesis and discuss some issues related to it. In Section 4, we give a characterisation of Lagrangian systems under the stochastisation hypothesis using PDEs of the Schrödinger type. Section 5 describes two applications of this formalism : In Section 5.1 we apply the stochastisation hypothesis in the study of the dynamics of a protoplanetary nebula. This work is related to the approach of S. Albeverio, Ph. Blanchard and R. Hoegh-Krohn ([3],[4]), L. Nottale ([41],[42],[45]) and J. Laskar $([\mathbf{2 8}],[\mathbf{2 9}])$ to the Titius-Bode law. We then apply the stochastisation hypothesis to the long term behaviour of the solar systems using the work of J. Laskar ([28]) in Section 5.2. Both of these applications give an analogue of the Titius-Bode law for the repartition of planetary distances around a star although the domain of application is different.

\section{Reminder about stochastic embedding}

We refer to [10] for a complete introduction to the stochastic embedding formalism for ordinary differential equations and to [9] for an overview. The formalism of embedding is described in $([\mathbf{1 2}],[\mathbf{1 3}])$ in full generality.

2.1. Notations. - Let $T>0, \nu>0$ and $d \in \mathbb{N}^{*}$. Let $\mathrm{L}$ be the set of all measurable functions $f:[0, T] \times \mathbb{R}^{d} \rightarrow \mathbb{R}^{d}$ satisfying the following hypothesis: There exists $K>0$ such that for all $x, y \in \mathbb{R}^{d}: \sup _{t}|f(t, x)-f(t, y)| \leq K|x-y|$ and $\sup _{t}|f(t, x)| \leq K(1+|x|)$.

We are given a probability space $(\Omega, \mathcal{A}, \mathbb{P})$ on which a family $\left(W^{(b, \sigma)}\right)_{(b, \sigma) \in \mathrm{L} \times \mathrm{L}}$ of Brownian motions indexed by $\mathrm{L} \times \mathrm{L}$ is defined. If $b, \sigma \in \mathrm{L}$, we denote by $\mathcal{P}^{(b, \sigma)}$ the natural filtration associated to $W^{(b, \sigma)}$. Let $\mathcal{P}$ be the filtration generated by the filtrations $\mathcal{P}^{(b, \sigma)}$ where $(b, \sigma) \in$ 
$\mathrm{L} \times \mathrm{L}$, and we set: For $t \in[0, T]$,

$$
\mathcal{P}_{t}=\bigvee_{(b, \sigma) \in \mathrm{L} \times \mathrm{L}} \mathcal{P}_{t}^{(b, \sigma)}
$$

Let $F\left([0, T] \times \mathbb{R}^{d}\right)$ be the space of measurable functions defined on $[0, T] \times \mathbb{R}^{d}$ and let $F([0, T] \times \Omega)$ be the space of measurable stochastic processes defined on $[0, T] \times \Omega$.

Let us define the involution $\phi: F\left([0, T] \times \mathbb{R}^{d}\right) \rightarrow F\left([0, T] \times \mathbb{R}^{d}\right)$ such that for all $t \in[0, T]$ and $x \in \mathbb{R}^{d},(\phi u)(t, x)=-u(T-t, x)$. We also define the time-reversal involution on stochastic processes: $r: F([0, T] \times \Omega) \rightarrow F([0, T] \times \Omega), r(X)_{t}(\omega)=X_{T-t}(\omega)$.

It is convenient to use the bar symbol to denote these two involutions. We now agree to denote deterministic functions by small letters and stochastic processes by capital letters. So there will not be any confusion when using the bar symbol: $\bar{u}:=\phi u$ and $\bar{X}:=r(X)$.

\subsection{Stochastic derivatives. -}

Definition 1. - We denote by $\Lambda^{1}$ the space of all diffusions $X$ satisfying the following conditions:

(i) $X$ is a solution on $[0, T]$ of the $S D E$ : $d X_{t}=b\left(t, X_{t}\right) d t+\sigma\left(t, X_{t}\right) d W_{t}^{(b, \sigma)}, \quad X_{0}=X^{0}$ where $X^{0} \in L^{2}(\Omega)$ and $(b, \sigma) \in \mathrm{L} \times \mathrm{L}$,

(ii) For all $t \in(0, T), X_{t}$ admits a density $p_{t}(\cdot)$,

(iii) Setting $a^{i j}=\left(\sigma \sigma^{*}\right)^{i j}$, for all $i \in\{1, \cdots, n\}, t_{0}>0$,

$$
\int_{t_{0}}^{T} \int_{\mathbb{R}^{d}}\left|\partial_{j}\left(a^{i j}(t, x) p_{t}(x)\right)\right| d x d t<+\infty
$$

(iv) For all $i, j, t$,

$$
\frac{\partial_{j}\left(a^{i j}(t, \cdot) p_{t}(\cdot)\right)}{p_{t}(\cdot)} \in \mathrm{L}
$$

A diffusion verifying (i) will be called a $(b, \sigma)$-diffusion and we denote it by $X^{(b, \sigma)}$.

We denote by $\Lambda_{v}^{1}$ the closure of $\operatorname{Vect}\left(\Lambda^{1}\right)$ in $L^{1}(\Omega \times[0, T])$ endowed with the usual norm $\|\cdot\|=E \int|\cdot|$.

We know that the reversed process of any element $\Lambda^{1}$ is still a Brownian diffusion driven by a Brownian motion $\widehat{W}^{(b, \sigma)}(c f[\mathbf{3 4}])$. We denote by $\widehat{\mathcal{P}}^{(b, \sigma)}$ the natural filtration associated to $\widehat{W}^{(b, \sigma)}$. Let $\widehat{\mathcal{P}}$ be the filtration defined by: For all $t \in[0, T]$,

$$
\widehat{\mathcal{P}}_{t}=\bigvee_{(b, \sigma) \in \mathrm{L} \times \mathrm{L}} \widehat{\mathcal{P}}_{t}^{(b, \sigma)} .
$$

We finally consider the filtration $\mathcal{F}$ such that $\mathcal{F}_{t}=\widehat{\mathcal{P}}_{T-t}$ for all $t \in[0, T]$.

\section{Proposition 1 (Definition of Nelson Stochastic derivatives)}


Let $X=X^{b, \sigma} \in \Lambda^{1}$ with $a^{i j}=\left(\sigma \sigma^{*}\right)^{i j}$ and $a^{j}=\left(a^{1 j}, \cdots, a^{d j}\right)$. For almost all $t \in(0, T)$, the Nelson stochastic derivatives exist in $L^{2}(\Omega)$ :

$$
\begin{aligned}
\text { (1) } D X_{t}: & =\lim _{h \rightarrow 0^{+}} E\left[\frac{X_{t+h}-X_{t}}{h} \mid \mathcal{P}_{t}\right]=b\left(t, X_{t}\right) \\
\text { (2) } \quad D_{*} X_{t}: & =\lim _{h \rightarrow 0^{+}} E\left[\frac{X_{t}-X_{t-h}}{h} \mid \mathcal{F}_{t}\right]=b\left(t, X_{t}\right)-\frac{1}{p_{t}\left(X_{t}\right)} \sum_{j} \partial_{j}\left(a^{j}\left(t, X_{t}\right) p_{t}\left(X_{t}\right)\right) .
\end{aligned}
$$

Therefore, for $X^{b, \sigma} \in \Lambda^{1}$, there exists a measurable function $b_{*}$ such that $D_{*} X_{t}=b_{*}\left(t, X_{t}\right)$. We call it the left velocity field of $X$. It turns out to be an important objet for the sequel. Also, this field is related to the drift of the time reversed process $\bar{X}$ through the following identity:

$$
D \bar{X}=-\overline{b_{*}} \text {. }
$$

We denote by $\Lambda^{2}=\left\{X \in \Lambda^{1} ; D X, D_{*} X \in \Lambda^{1}\right\}$ and $\Lambda_{v}^{2}$ the closure of $\operatorname{Vect}\left(\Lambda^{2}\right)$ in $L^{1}(\Omega \times$ $[0, T])$. We then define $\Lambda_{v}^{k}$ in an obvious way.

We denote by $\mathcal{D}_{\mu}$ the stochastic derivative introduced in ([9] Lemme 1.2) and defined by

$$
\mathcal{D}_{\mu}=\frac{D+D_{*}}{2}+\mu \frac{D-D_{*}}{2}, \quad \mu \in\{0, \pm 1, \pm i\} .
$$

We can extend $\mathcal{D}$ by $\mathbb{C}$-linearity to complex processes $\Lambda_{\mathbb{C}}^{1}:=\Lambda_{v}^{1} \oplus i \Lambda_{v}^{1}$.

Remark 1. - We denote by $\pi: C^{0} \rightarrow \Lambda^{0}$ the mapping defined by $\pi(x)_{t}(\omega)=x(t)$ for all $\omega \in \Omega$ and $t \in \mathbb{R}$. If $x \in C^{1}$, we have

$$
\mathcal{D}_{\mu} \pi(x)_{t}=\pi\left(\frac{d x}{d t}\right) .
$$

In this sense, the stochastic derivative is an extension of the classical derivative as it reduces to the classical one on $\pi\left(C^{1}\right)$.

Theorem 1. - Let $X^{(b, \sigma)} \in \Lambda^{1}, a=\sigma \sigma^{*}$ and $f \in C^{1,2}\left(I \times \mathbb{R}^{d}\right)$ such that $\partial_{t} f, \nabla f$ and $\partial_{i j} f$ are bounded. We get:

$$
\mathcal{D}_{\mu} f\left(t, X_{t}^{(b, \sigma)}\right)=\left(\partial_{t} f+\mathcal{D} X_{t}^{(b, \sigma)} \cdot \nabla f+\frac{\mu}{2} \sum_{k, j} a^{k j} \partial_{k j} f\right)\left(t, X_{t}^{(b, \sigma)}\right) .
$$

Moreover, we can generalize for the operator $\mathcal{D}_{\mu}$ the "product rule" given by Nelson in [38] p.80, which is a fundamental tool to develop a stochastic calculus of variation:

Lemma 1. - Let $X, Y \in \Lambda_{\mathbb{C}}^{1}$. Then $E\left[\mathcal{D}_{\mu} X_{t} \cdot Y_{t}+X_{t} \cdot \mathcal{D}_{-\mu} Y_{t}\right]=\frac{d}{d t} E\left[X_{t} \cdot Y_{t}\right]$.

The proof is an immediate consequence of the form of the operator $\mathcal{D}$ and the fact that $\Lambda^{1}$ is a subspace of the class $S(\mathcal{F}, \mathcal{G})([62]$ p.226) for which W. Zheng and P-A. Meyer show the product rule of Nelson ( $c f[\mathbf{6 2}]$ Th. I.2 p.227). 
We denote by $\Sigma_{\sigma}$ the set of all Brownian diffusions in $\Lambda^{1}$ such that $X_{0} \in L^{2}(\Omega), \sigma$ is a constant. We set

$$
\Sigma=\bigcup_{\sigma \in \mathbb{R}} \Sigma_{\sigma}
$$

We finally denote by $\Sigma_{\sigma}^{\nabla}$ (resp. $\Sigma^{\nabla}$ ) the subspace of $\Sigma_{\sigma}$ (resp. $\Sigma$ ) of all Brownian diffusions with a gradient drift.

2.3. Stochastic embedding. - The formalism of embedding is developed in [10]. It provides a way to extend a given ordinary differential equation over the set of stochastic processes. This extension is different from the usual point of view of stochastic differential equations introduced by K. Itô [26]. Here, we give a definition for a special subclass of ordinary differential equations which covers the applications of Section 5 and we refer to [10] for a general framework.

Let $x_{t}$ be a solution of the ordinary differential equation

$$
\frac{d^{2} x_{t}}{d t}=-\nabla U\left(x_{t}\right)
$$

that we call Newton's equation in the following. This equation can be derived from a variational principle called the least action principle in Physics (see [2]). Precisely, let us denoted by $\mathcal{L}$ the Lagrangian functional defined by

$$
\mathcal{L}\left(x_{t}\right)=\int_{a}^{b}\left(\frac{1}{2}\left(\frac{d x_{s}}{d s}\right)^{2}-U\left(x_{s}\right)\right) d s,
$$

for all $x_{t} \in C^{2}\left([a, b], \mathbb{R}^{d}\right)$. The solutions of the Newton's equation (7) coincide with the critical points of $\mathcal{L}$, i.e. that $x_{t}$ is a solution of (7) if and only if $x_{t}$ satisfies $\mathcal{D} \mathcal{L}\left(x_{t}\right)\left(h_{t}\right)=0$ for all $h_{t}$ such that $h_{a}=h_{b}=0$, where $\mathcal{D} \mathcal{L}\left(x_{t}\right)\left(h_{t}\right)$ denotes the Frechet derivatives of $\mathcal{L}$ in $x_{t}$ along the direction $h_{t}$.

We can generalize the Newton's equation using (at least ${ }^{(1)}$ ) two different strategies : the differential and the variational one.

2.3.1. Stochastic differential embedding. - The differential embedding of the Newton's equation is based on the differential operator formulation of the equation. We denote by $P(x)$ the symbol of the differential operator associated to (7) and defined by

$$
P(z)=z^{2}+\nabla U(x) .
$$

\footnotetext{
${ }^{(1)}$ The formalism of embedding defined in [13] which is not restricted to stochastic processes, covers in fact three different ways of generalizing a differential or partial differential equation : the differential, variational and integral one. Each of them are based on the three common ways of expressing an equation. We refer to [13] for more details and examples.
} 
The Newton's equation is then given by

$$
P\left(\frac{d}{d t}\right) \cdot x_{t}=0
$$

We can defined the differential operator on the set of stochastic processes as long as we have a notion of derivative and functional.

Let $a: \mathbb{R}^{d} \rightarrow \mathbb{R}^{m}$ be a function. A natural extension of $a$ over stochastic processes is given by

$$
a\left(X_{t}\right)(\omega)=a\left(X_{t}(\omega)\right),
$$

for all $\omega \in \Omega$. Using this definition, we can define the following stochastic version of the Newton's equation :

Definition 2. - The stochastic differential embedding of equation (10) is defined by for all $X_{t} \in \Lambda^{3}$ by

$$
P\left(\mathcal{D}_{\mu}\right) \cdot X_{t}=0
$$

The previous construction can be generalized to arbitrary differential operators. We refer to [10] for more details.

Remark 2. - The principle of taking the symbol of a differential operator and replacing the classical derivative by a new one is a well-known procedure in PDEs when dealing with PDEs in the sense of Schwartz's distribution theory (see [56]). In this sense, the generalization of a classical PDE to Schwartz's distributions is a particular example of a differential embedding (see [12] and [13]).

Remark 3. - A main property of this stochastic extension of a differential equation is that it reduces to the classical equation over the set $\pi\left(C^{n}\right)$ where $n$ is the order of the differential operator. This remark justifies also the terminology of embedding : the initial equation is contained in the extended one. In particular, this stochastic extension does not coincide with the classical theory of stochastic differential equations due to K. Ito. This comes from the fact that Ito's strategy does not use the differential formulation of a differential equation as a starting point but the integral formulation thus providing a different theory.

As a consequence, the embedded Newton's equation is given by

$$
\mathcal{D}_{\mu}^{2} X_{t}=-\nabla U\left(X_{t}\right)
$$

Particular cases of this equation have been studied by E. Nelson [38] and Thieullen-Zambrini [52] for the case $\mu=i$ (see [52], Prop.2.7). It must be noted that we have just defined the stochastic analogue of the classical derivative in order to generalize our differential equation, 
i.e. when dealing with a given stochastic process, only a stochastic notion of speed is given. In particular, no definition of a stochastic acceleration is needed in order to derive this equation as its form is fixed by the stochastic differential embedding.

2.3.2. Stochastic analogue of dynamical quantities : speed and acceleration. - The two basic quantities allowing to write the fundamental law of dynamics for forces deriving from a potential $U$

$$
a=-\nabla U(x),
$$

is the notion of speed $v$ and acceleration $a$ for a particle. If $x(t)$ denotes the motion of the particle, we have by definition

$$
v=\frac{d x}{d t} \quad a=\frac{d^{2} x}{d t^{2}} .
$$

For a stochastic process $X$ the natural analogues are given by

$$
\mathcal{V}=\mathcal{D} X, \quad \mathcal{A}=\mathcal{D}^{2} X
$$

As the definition of the stochastic speed is fixed, i.e. when the real number $\mu$ is given, then the notion of stochastic acceleration is also fixed by substitution of $\mathcal{D}$ instead of $d / d t$. This definition for a stochastic acceleration follows then from the differential stochastic embedding procedure, i.e. by a pure algebraic procedure.

Remark 4. - In ([38], p.81-82) E. Nelson introduces a definition of a stochastic acceleration which does not follows from the definition of a stochastic speed as in our case using the stochastic differential embedding. Indeed, Nelson looks for expressions involving both the backward anf forward Nelson's derivatives which are quadratic. He then makes some assumptions in order to select a definition of acceleration which is in agreement with what we are waiting for in specific stochastic systems. We refer to ([10], Remark 1.4, p.13-14) for more details.

Definition 16 is interesting for two reasons :

- This choice keeps the coherence between a direct stochastic analogue of the fundamental law of dynamics which can be written

$$
\mathcal{A}=-\nabla U(X),
$$

and the differential stochastic embedding defined in the previous Section. As a consequence, we can formulate a direct stochastic analogue of the laws of dynamics.

- We can easily compute

$$
\mathcal{D}_{i}^{2}=\frac{D D_{*}+D_{*} D}{2}+i \frac{D^{2}-D_{*}^{2}}{2} .
$$


The real part of $\mathcal{D}^{2}$ coincides with the notion of mean acceleration introduced by Nelson [38] in his dynamical theory of the Brownian motion, for which he had conjectured that it is the more relevant quantity describing an acceleration on Brownian diffusions.

Remark 5. - The previous remark answers the objection of L. Nottale [45], p. 385 about the work of S. Albeverio and al. [3]. They have used the theory of Nelson about stochastic mechanics [38] to write the equations of motion of a particle in a protoplanetary nebula (see also Section 5.1). E. Nelson [38] as well as S. Albeverio and al. ([3], p. 367) have to postulate the form of the mean acceleration. This is not the case in the present framework as the analogue of the acceleration is fixed by the stochastic embedding to be $\mathcal{D}^{2}$.

2.3.3. Stochastic variational embedding. - Another point of view is to define the stochastic analogue of equation (7) using the variational characterization. The Lagrangian functional $\mathcal{L}$ can be extended to stochastic processes easily. Indeed, we first remark that the quantity $\frac{1}{2}\left(\frac{d x_{s}}{d s}\right)^{2}-U\left(x_{s}\right)$ keep sense over stochastic processes replacing $\frac{d}{d t}$ by the stochastic derivative $\mathcal{D}_{\mu}$. We then define the following stochastic analogue of the Lagrangian functional (8) :

Definition 3. - The stochastic embedded version of the Lagrangian functional $\mathcal{L}$ defined by (8) denoted by $\mathcal{L}_{\text {stoc }}$ is given for all $X_{t} \in \Lambda^{3}$ by

$$
\mathcal{L}_{\text {stoc }}\left(X_{t}\right)=\mathrm{E}\left[\int_{a}^{b}\left(\frac{1}{2} \mathcal{D}_{\mu}^{2} X_{s}-U\left(X_{s}\right)\right) d s\right] .
$$

Remark 6. - More generally, if $L(t, x, v)$ denotes a Lagrangian function over $\mathbb{R} \times \mathbb{R}^{d} \times \mathbb{R}^{d}$ and

$$
\mathcal{L}(x)=\int_{a}^{b} L(s, x(s), d x / d t)(s) d s
$$

the associated Lagrangian functional, the stochastic analogue is given by

$$
\mathcal{L}_{\text {stoc }}\left(X_{t}\right)=\mathrm{E}\left[\int_{a}^{b} L\left(s, X_{s}, \mathcal{D}_{\mu} X_{s}\right) d s\right] .
$$

The form of the functional is then fixed by the formalism of stochastic embedding.

It is natural to look for the Frechet derivative of $\mathcal{L}_{\text {stoc }}$ in a direction $H_{t} \in V \subset \Lambda^{1}$ denoted by $D \mathcal{L}_{\text {stoc }}\left(X_{t}\right)\left(H_{t}\right)$. The set $V$ is the set of variations. We use the following terminology :

Definition 4. - A process $X_{t} \in \Lambda^{3}$ is a $V$-critical process of the functional $\mathcal{L}_{\text {stoc }}$ if $D \mathcal{L}_{\text {stoc }}\left(X_{t}\right)\left(H_{t}\right)=0$ for all $H_{t} \in V$.

Using ([10], Thm.3.1 p.33) we have the following theorem : 
Theorem 2. - A necessary and sufficient condition for a process $X \in \Lambda^{3}$ to be a $\Lambda^{1}$-critical process of the functional $\mathcal{L}_{\text {stoc }}$ is that it satisfies

$$
\mathcal{D}_{-\mu} \mathcal{D}_{\mu} X_{t}=-\nabla U\left(X_{t}\right)
$$

The stochastic embedded Newton's equation coincides with (22) only when $\mu=0$.

However, if we change the set of variations using the space of Nelson differentiable processes:

$$
\mathcal{N}^{1}=\left\{X \in \Lambda^{1}, D X=D_{*} X\right\} .
$$

We obtain the following partial result (see [10], Lemma 3.4 p.34) :

Proposition 2. - A solution of the equation

$$
\mathcal{D}_{\mu}^{2} X_{t}=-\nabla U\left(X_{t}\right),
$$

is a $\mathcal{N}^{1}$-critical process for the functional $\mathcal{L}_{\text {stoc }}$

We have not been able to prove the converse of this lemma for $\mathcal{N}^{1}$-variations.

2.4. Coherence of stochastic embedding. - The two previous approaches to deal with an extension of a given ordinary differential equation over stochastic processes are natural. However, as the least-action principle is a first principle of Physics, it is certainly preferable to keep track of this structure in the stochastic case. The coherence problem introduced in $[\mathbf{1 0}]$ is to determine when the differential and variational stochastic embedding coincide. The previous results can be summarized in the following Theorem :

Theorem 3. - The differential and variational embedding of the Newton's equation coincide if and only if $\mu=0$.

If $\mu \neq 0$ we have only a partial result, as the stochastic Newton's equation does not characterize the critical point of the stochastic functional $\mathcal{L}_{\text {stoc }}$ over $\mathcal{N}^{1}$.

2.5. Lifting of symmetries and a stochastic Noether theorem. - In the two previous Sections, we have discussed two distinct generalizations, differential and variational, of a given classical differential equation and in which case they provide the same extension to stochastic processes. The "natural" character of the generalized equation is based on the preservation of a given specific property : the algebro-differential structure in the case of the differential embedding and the fact to be a critical point of a functional in the variational case. Of course, many other properties of a given equation are important and suitable to be preserved. For instance, symmetries play an important role in the dynamics as they induce the existence of first integrals by the classical Noether theorem (see for example [2], p.88). First integrals 
constrain the behaviour of the solutions of the equation. A natural demand is then to preserve as far as possible the symmetries of the initial system under an embedding.

This problem was discussed in ([10] and [11]) in the context of the stochastic embedding of Lagrangian systems. A related but different approach is given by Thieullen and Zambrini in [53]. We give here a short account of these results.

2.5.1. First integrals of stochastic dynamical systems. - A definition of first integrals for stochastic dynamical systems obtained by the stochastic embedding is the following (see [11], Definition 4.4, p. 263) :

Definition 5. - Let $X \in \Lambda^{1}$ be a solution of the stochastic Newton's equation. The map $I: \Lambda^{1} \rightarrow C^{0}(\mathbb{R}, \mathbb{C})$ is a first integral for $X$ if $\frac{d}{d t} I(X)_{t}=0$.

Other definitions of first integrals can be given. We refer to ([11], p.262-263 and [10], §.7, p. 39-40) for a discussion, in particular a comparison with Thieullen-Zambrini [53].

2.5.2. Stochastic lifting of symmetries. - A stochastic embedding provided a natural stochastic counter part to classical functions. As a consequence, we obtain the following stochastic lifted notion of one parameter group of diffeomorphisms.

Definition 6. - Let $\phi: \mathbb{R}^{d} \rightarrow \mathbb{R}^{d}$ be a diffeomorphism. The stochastic suspension of $\phi$ is the mapping $\phi^{\text {stoc }}: \Lambda^{0} \rightarrow \Lambda^{0}$ defined for all $X \in \Lambda^{0}$ by $\phi^{\text {stoc }}(X)_{t}(\omega)=\phi\left(X_{t}(\omega)\right)$. We denote by $\Phi=\left\{\phi_{s}\right\}_{s \in \mathbb{R}}$ a one parameter group of diffeomorphisms $\phi_{s}: \mathbb{R}^{d} \longrightarrow \mathbb{R}^{d}$. The associated stochastic lifted group of diffeomorphisms $\Phi_{\mathrm{stoc}}$ is defined by $\left\{\phi_{s}^{\mathrm{stoc}}\right\}_{s \in \mathbb{R}}$.

A one parameter group of diffeomorphisms $\Phi=\left\{\phi_{s}\right\}_{s \in \mathbb{R}}$ is said to be admissible if for all $s \in \mathbb{R}, \phi_{s} \in C^{2},(s, x) \rightarrow \partial_{x} \phi_{s}(x) \in C^{3}$ and formula (6) is valid for all $f=\phi_{s}, s \in \mathbb{R}$.

2.5.3. Stochastic invariance. - A stochastic Lagrangian functional is said to be invariant under a stochastic lifted one parameter group of diffeomorphisms $\Phi_{\text {stoc }}$ if for all $X \in \Lambda^{1}$, we have $L\left(\phi_{s}^{\text {stoc }}(X), \mathcal{D}\left(\phi_{s}^{\text {stoc }}(X)\right)\right)=L(X, \mathcal{D})$.

The notion of invariance introduced in Thieullen-Zambrini (see [53], p.313) can be formulated using our notion of stochastic lifted group of diffeomorphisms. The stochastic invariance defined by Yasue (see [60], p.332, formula (3.1)) is different. We refer to ([10], Remark 3.3, p.37) for more details.

A natural question in the embedding formalism is to know whether or not a group of symmetries is preserved under the stochastic embedding : 
Problem 1 (Persistence of invariance). - Assume that a Lagrangian L is invariant under a group of symmetries $\left\{\phi_{s}\right\}_{s \in \mathbb{R}}$. Do we have the stochastic invariance of the Lagrangian $L$ under the stochastic lifted one parameter group of diffeomorphisms $\left\{\phi_{s}^{\text {stoc }}\right\}_{s \in \mathbb{R}}$ ?

A general solution of this problem is out of reach for the moment. In the following, we give a partial solution to this problem following our previous approach to the non-differentiable case $[\mathbf{1 4}]$.

Definition 7 (Strong invariance). - Let $\Phi=\left\{\phi_{s}\right\}_{s \in \mathbb{R}}$ be a one parameter group of diffeomorphisms. An admissible Lagrangian $L$ is said to be strongly invariant under the action of $\Phi$ if

$$
L(t, x, v)=L\left(t, \phi_{s}(x), \phi_{s}(v)\right), \quad \forall s \in \mathbb{R}, \forall t \in I, \forall x \in \mathbb{R}^{d}, \forall v \in \mathbb{R}^{d} .
$$

As an example we can consider the following Lagrangian $L$, given by:

$$
L(t, x, v)=\frac{1}{2}\|v\|^{2}-\frac{1}{\|x\|^{2}}
$$

If $\phi_{s}$ is a rotation, $\phi_{s}(x):=e^{i s \theta} x$, then the Lagrangian $L$ is strongly invariant.

The main property that the stochastic lifted group of diffeomorphisms must satisfy is the following commutation property :

Definition 8 (D-commutation). - Let $\Phi=\left\{\phi_{s}\right\}_{s \in \mathbb{R}}$ be a one parameter group of diffeomorphisms. We say that the associated stochastic lifted group satisfies the $\mathcal{D}$-commutation property, if

$$
\mathcal{D}\left(\phi_{s}^{\text {stoc }}(X)_{t}\right)=\phi_{s}^{\text {stoc }}\left(\mathcal{D}(X)_{t}\right), \quad \forall s \in \mathbb{R}, \quad \forall t \in \mathbb{R} .
$$

Using these two notions, we have the following sufficient condition for persistence of invariance under stochastisation :

Theorem 4. - Let $L$ be an admissible Lagrangian system, strongly invariant under a one parameter group of diffeomorphisms $\Phi=\left\{\phi_{s}\right\}_{s \in \mathbb{R}}$ satisfying the commutation property. Then, $L$ is invariant under the stochastic lifted group of diffeomorphisms $\left\{\phi_{s}^{\text {stoc }}\right\}_{s \in \mathbb{R}}$.

Proof of Theorem 4. - Using the commutation property given by equation 25, we have

$$
L\left(\phi_{s}^{\text {stoc }} X, \mathcal{D}\left(\phi_{s}^{\text {stoc }} X\right)\right)=L\left(\phi_{s}^{\text {stoc }} X, \phi_{s}^{\text {stoc }}(\mathcal{D} X)\right) .
$$

By the strong invariance property, we obtain

$$
L\left(\phi_{s}\left(X_{t}(\omega)\right), \phi_{s}\left((\mathcal{D} X)_{t}(\omega)\right)\right)=L\left(X_{t}(\omega),(\mathcal{D} X)_{t}(\omega)\right), \quad \forall t \in I, \quad \omega \in \Omega .
$$

This concludes the proof. 
The commutation property is satisfied by linear maps whose matrix coefficients do not depend on the time variable $t$, as for example rotations or translations.

2.5.4. Stochastic Noether's theorem. - In [10] we prove the following version of a stochastic Noether's theorem :

Theorem 5. - Let $L$ be an admissible lagrangian with all second derivatives bounded, and invariant under a stochastic lifted one-parameter group of diffeomorphisms $\Phi_{\text {stoc }}=\left\{\phi_{s}^{\text {stoc }}\right\}_{s \in \mathbb{R}}$. Let $\mathcal{L}$ be the associated functional defined by (21) on $\Xi \cap \Lambda^{1}$. Let $X \in \Xi \cap \Lambda^{1}$ be a $\Lambda^{1}$-critical point of $\mathcal{L}$. Then, we have

$$
\frac{d}{d t} E\left[\left.\partial_{v} L \cdot \frac{\partial Y}{\partial s}\right|_{s=0}\right]=0
$$

where

$$
Y_{s}=\Phi_{s}(X)
$$

We refer to $([\mathbf{1 0}], p .38)$ for the proof.

2.5.5. An example : rotations and stochastisation. - The previous theorem can be applied in the following situation : We consider the Lagrangian system defined by

$$
L(t, x, v)=\frac{1}{2}\|v\|^{2}-\frac{1}{\|x\|^{2}}
$$

We have already seen that $L$ is strongly invariant under the group $\phi_{s}$ of rotations, $\phi_{s}(x):=$ $e^{i s \theta} x$. As the $\phi_{s}$ are linear maps whose matrix coefficients do not depend on $t$, the commutation property is easily satisfied for this group. As a consequence, the invariance of the initial Lagrangian system under rotations is preserved under stochastisation.

The first integrals associated to rotations in this system correspond to the components of the angular momentum. The previous result implies that such quantities are also preserved by stochastisation.

\section{The Stochastisation hypothesis}

In this Section using the previous stochastic embedding formalism, we state the stochastisation hypothesis for a physical system. The strategy of the stochastic embedding can be extended over arbitrary stochastic processes. However, our first attempt in Section 2 deals with diffusion processes. In Section 3.3 we discuss this assumption. We prove in particular that under general regularity assumptions the set of stochastic processes is the set of diffusion processes. 
3.1. The stochastisation hypothesis. - The stochastisation hypothesis is a way to define a stochastic extension of a given ordinary differential equation. The previous section has given two natural extensions of ordinary differential equations : the differential and the variational one.

- The stochastic differential embedding of an equation keeps the algebraic structure of the equation in term of operators. As the differential operator codes the different dynamical effects, the conservation of this structure is by itself suitable.

- The stochastic variational embedding is based on a first principle of Physics : the leastaction principle. A main property of this characterization is that it does not depend on the coordinates system on the contrary to the form of the differential operator.

As a consequence, a stochastic extension of a given ordinary differential equation must be coherent in order to preserve both the dynamical and physical background of the equation. The two results of Section 2.4 give a strong support to the $\mu=0$ case. In any cases, Proposition 2 ensures that the stochastic differential embedding preserves in a weak sense the least-action principle. The main principle of our extension can be summarized in the following diagram :

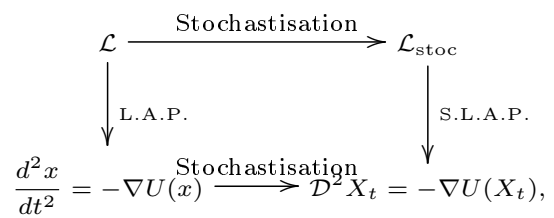

where the acronyms L.A.P. and S.L.A.P. are for Least Action Principle and Stochastic Least Action Principle in the sense of Proposition 2. The word stochastisation means that we apply the stochastic embedding.

The Stochastisation hypothesis can now be formulated :

Stochastisation Hypothesis : Let $x_{t}$ be a physical process governed by a given ordinary differential equation. The stochastic extension of this equation is given by the differential stochastic embedding of the equation.

There exist already many ways of generalizing differential equations over stochastic processes, the most common one being to add a noise to the equation in integral form and to interpret the corresponding object using K. Itô theory [26] of stochastic differential equations. However, no analogue of the coherence between the stochastic dynamical/variational formulations of a given equation has been formulated in this case. 
3.2. Discussion of the stochastisation hypothesis. - In this Section, I would like to make only few comments on the physical background underlying the stochastisation hypothesis. This discussion is informal. However, it offers some ideas explaining the choice we have made in the formulation of the stochastisation hypothesis.

In the stochastisation hypothesis a specific role is given to the underlying classical differential equation representing the motion or behaviour of a quantity under the law of Physics. The formulation of a physical law is made using a given mathematical framework. The mathematical framework by itself possesses constrains. For example, classical mechanics is formulated using the classical differential calculus. However, the underlying law applies by definition to the real world. In the real world the limitations imposed by the mathematical framework do not need to be satisfied. However, even if the law applies to (a priori) very complicated (at least sufficiently in order to describe the underlying motion) quantities (in our setting for example : the motion of a particle must be described by a stochastic process and not by a smooth function), the only way to have access to this law is to first check the formulation in very particular cases where the behaviour is very regular. The classical behaviours are then understood as good conditions under which the form of the laws of Nature can be found, or in other words, the form of the fundamental equations associated to these laws.

The fact that the form of the equation is meaningful can be seen as a consequence of an extended form of the relativity principle in Physics which can be roughly formulated as follows : equations of physics must keep the same forms under admissible family of transformations. This is for example explicitly used by Nottale ([40], p.195-196) in his approach to quantum mechanics via the scale relativity theory. The characterisation of the admissible set of transformations is of course not always easy. We refer to the classical work of Levy-Leblond [32] for the classical case of special relativity and to [8] for the scale relativity case following the idea of Nottale [40].

Naturally, all these points and arguments can be discussed and improved. However, I believe that they can be useful to construct a meaningful formalism for the laws of physics over stochastic processes. The previous point of view is also related to Prigogine approach to chaotic dynamical systems as exposed for example in his book "The laws of Chaos" [47].

3.3. Stochastic processes and stochastisation : diffusion processes ?- In this Section, we prove that natural regularity conditions on the process $X$ that we must characterize to model a given system yield to the structure of diffusion. 
The hypothesis that $X$ has continuous paths and is a Markov process are relevant for many physical systems. To go further, one can add a first order regularity condition for the averages of suitable functionals of $X$ : Namely the quantity

$$
\frac{E^{x} f\left(X_{t}\right)-f(x)}{t}
$$

converges for all $x \in \mathbb{R}^{d}$ and all $f \in C_{0}^{\infty}\left(\mathbb{R}^{d}\right)$ when $t \downarrow 0$. As pointed out in [25] e.g., one obtains the existence of a drift coefficient $b$ and a covariance matrix $a$ :

$$
\begin{aligned}
\lim _{t \downarrow 0} \frac{E^{x}\left[X_{t}-x\right]}{t} & =b(x) \\
\lim _{t \downarrow 0} \frac{E^{x}\left[\left(X_{t}-x\right)^{t}\left(X_{t}-x\right)\right]}{t} & =a(x) .
\end{aligned}
$$

These equalities are the starting point of diffusion theory. See Definition 1.1 p.282 in [25] or Definition 1.2 p.110 in [55]. A precise theorem is given in [38] p.77 concerning the structure of $X$ when assuming the conditions (31) and (32) for a Markov process. Then $X$ has to solve a stochastic differential equation. The relationship between diffusions and solutions of stochastic differential equations is one of the crucial points of Ito's Theory. We refer to Chap.V in [25] or Chap.5 in [55] regarding the involved results.

In our framework, we have to point out that the hypothesis of choosing Brownian diffusions arises from regularity conditions on the process. These conditions can be seen as a first requirement to write an embedded equation; Particularly, the condition (31) ensures the existence of the first order stochastic derivative of $X$ with respect to its present. Moreover, the drift $b$ will be unknown in an embedded equation.

\section{The stochastisation hypothesis and the Newton's equation}

We discuss the application of the stochastisation hypothesis for the Newton's equation. In this case, depending on the assumption about the underlying set of stochastic processes, we provide a characterisation of the solutions of the associated stochastic Lagrangian systems using PDEs of the Schrödinger type.

4.1. The stochastic Newton equation. - In [10] we have proved that the solutions of the stochastic Newton's equation (13) possess a density $p_{t}$ satisfying the linear Schrödinger equation as long as we restrict our attention on diffusions with gradient drift. Using the characterization of gradient drift diffusions obtained by S. Darses and I. Nourdin [17], we can cancel this restriction. 
Let $\Psi(t, x)$ be the solution of the Schrödinger equation with initial condition $\Psi_{0}$ :

$$
\left\{\begin{array}{ccc}
i \sigma^{2} \partial_{t} \Psi+\frac{\sigma^{4}}{2} \Delta \Psi & =U \Psi \\
\Psi(0, \cdot) & =\Psi_{0}
\end{array}\right.
$$

Theorem 6. - Set $\sigma \neq 0$. Let $X^{0}$ be a random variable with density $\left|\Psi_{0}\right|^{2}$. Then a solution $X \in \Lambda^{2}$ of the system

$$
\left\{\begin{array}{l}
\mathcal{D}^{2} X_{t}=-\nabla U\left(X_{t}\right) \\
X_{0}=X^{0}
\end{array}\right.
$$

is governed via $p_{t}(x)=|\Psi(t, x)|^{2}$ where $\Psi$ is a solution of the linear Schrödinger equation (33). It is the Nelson diffusion of the type

$$
d X_{t}=\left(\Re \frac{\nabla \Psi}{\Psi}+\Im \frac{\nabla \Psi}{\Psi}\right)\left(t, X_{t}\right) d t+\sigma d W_{t} .
$$

Following [10], we have only to prove that the stochastic Newton's equation implies that the diffusion has a gradient drift. This is precisely the content of (Theorem 5, p. 396 in [17]):

Theorem 7. - Let $X \in \Sigma_{\sigma}$. We then have the following equivalence:

$$
D^{2} X_{t}=D_{*}^{2} X_{t} \text { for almost all } t \in(0, T) \quad \Longleftrightarrow \quad X \in \Sigma_{\sigma}^{\nabla} \text {. }
$$

Moreover, the assumptions made by Carlen [7] ensure the existence of the Nelson diffusion (35) :

Let $U$ and $\Psi_{0}$ be functions satisfying the following hypothesis:

(i) $\Psi_{0}$ is a $C^{1}\left(\mathbb{R}^{d}, \mathbb{C}\right)$ function such that $\int_{\mathbb{R}^{d}}\left|\nabla \Psi_{0}(x)\right|^{2} d x<\infty$,

(ii) the function $U$ is a smooth Rellich class potential, i.e. the domain of $U$ (as a multiplication operator on $L^{2}\left(\mathbb{R}^{d}\right)$ ) contains $\operatorname{Dom}\left(-\frac{1}{2} \Delta\right)$ and there exists $a, b<1$ such that for all $\varphi \in \operatorname{Dom}\left(-\frac{1}{2} \Delta\right):\|U \varphi\| \leq a\left\|H_{0} \varphi\right\|+b\|\varphi\|$.

Remark 7. - The previous result justifies two important features of L. Nottale's approach ([40],[41],[43]) to quantization effect in the solar system and quantum mechanics using the principle of scale relativity :

- The Schrödinger equation discussed by L. Nottale in [40] in a different context and used for example in (443], equation (7), p. 1020) in his study of quantization of the solar system.

- The form of the Lagrangian functional (see [43], p. 1019).

Both of these objects are obtained directly as an application of the stochastic embedding framework. 
Remark 8. - In order to derive the previous equation in the context of the dynamics of a protoplanetary nebula, S. Albeverio and al. ([3], p.367) make an assumption of time symmetry in a stochastic sense which is

$$
-D X_{t}=D_{*} X_{t}
$$

This assumption is difficult to justify as collisions usually lead to irreversible systems via dissipation of energy. However, they noted that this equation can also be obtained under the assumption that the diffusions have gradient drift (see [3], Remark p. 368). In our setting, no assumption is made on the set of diffusion processes except that they satisfy the stochastic embedded Newton's equation. This equation induces a gradient drift structure on the diffusion processes which are solutions.

The previous derivation without the time symmetry assumption answers an objection made by L. Nottale (see [43] p.385) on the approach of S. Albeverio and al. [3]. In [43] the breaking of time reversibility is of fundamental importance.

4.2. The reversible stochastic Newton's equation. - Many physical problems possess a fundamental property called reversibility, i.e. that if $x_{t}$ is a solution of the equation then $x_{-t}$ is a solution of the same equation. This is for example the case of the Newton's equation. A natural demand is then to extend the equation over the set of stochastic processes conserving this property. Of course, the notion of reverse solution is not always easy to define and we prefer to use an algebraic characterization, saying that the operator is invariant under the substitution of $\frac{d}{d t}$ by $-\frac{d}{d t}$. In the framework of the stochastic derivatives introduced in Section 2.2, this substitution is the analogue of replacing $\mathcal{D}_{\mu}$ by $-\mathcal{D}_{-\mu}$. Using the definition of $\mathcal{D}_{\mu}$ it is easy to see that we preserve the reversibility property of the equation if and only if $\mu=0$.

We now consider the following equation

$$
\mathcal{D}_{0}^{2} X_{t}=-\nabla U\left(X_{t}\right)
$$

We want to characterize particular diffusion solutions of (38). We are interested in solutions having a gradient drift and belonging to $\Sigma_{\sigma}$. By making the same Nelson change of variables, we obtain that $\Psi$ has to solve a non-linear Schrodinger equation. We have to note that Misawa and Yasue [35] have already introduced the operator $\mathcal{D}_{0}$ when they want to put Nelson's stochastic mechanics into a lagrangian setting using $\mathcal{D}_{0}$. To this end, they modified the Newtonian potential by an "adequate" term. Here we can relate the equation (38) to a singular non linear Schrodinger equation: 
Theorem 8. - Let $X \in \Sigma_{\sigma}^{\nabla}$ be a gradient diffusion solution of (38). The density of this solution is governed via $p_{t}(x)=|\Psi(t, x)|^{2}$ where $\Psi$ is a solution of the nonlinear Schrödinger equation:

$$
i \sigma^{2} \partial_{t} \Psi+\sigma^{2} \frac{\Psi}{|\Psi|} \Delta|\Psi|+\frac{\sigma^{4}}{2} \Delta \Psi=U \Psi
$$

Proof. - Let us make again the change of variable $\Psi=e^{\frac{R+i S}{\sigma^{2}}}$ where

$$
\begin{aligned}
\nabla R & =\frac{\sigma^{2}}{2} \frac{\nabla p}{p}, \\
\nabla S & =b-\frac{\sigma^{2}}{2} \frac{\nabla p}{p}=\mathcal{D}_{0} X_{t} .
\end{aligned}
$$

So $\mathcal{D}_{0}^{2} X_{t}=\partial_{t} \nabla S+\left(\partial_{x} \nabla S\right) \nabla S$. We have:

$$
\sigma^{4} \frac{\Delta \Psi}{\Psi}=\sigma^{2}(\Delta R+i \Delta S)+2 i \nabla R \cdot \nabla S+|\nabla R|^{2}-|\nabla S|^{2} .
$$

Since $\nabla|\nabla S|^{2}=2\left(\partial_{x} \nabla S\right) \nabla S$ and $\nabla S=\sigma^{2} \Im \frac{\nabla \Psi}{\Psi}$, we can write

$$
\mathcal{D}_{0}^{2} X_{t}=-\sigma^{2} \Re\left(i \partial_{t} \frac{\nabla \Psi}{\Psi}+\frac{\sigma^{2}}{2} \nabla \frac{\Delta \Psi}{\Psi}-\nabla \Delta R-\nabla \frac{|\nabla R|^{2}}{\sigma^{2}}\right)
$$

But

$$
\begin{aligned}
\nabla \Delta R+\nabla \frac{|\nabla R|^{2}}{\sigma^{2}} & =\frac{\sigma^{2}}{2}\left(\Delta \frac{\nabla p}{p}+\left(\partial_{x} \frac{\nabla p}{p}\right) \frac{\nabla p}{p}\right) \\
\nabla \Delta R+\nabla \frac{|\nabla R|^{2}}{\sigma^{2}} & =\frac{\sigma^{2}}{2}\left(\Delta \frac{\nabla p}{p}+\left(\partial_{x} \frac{\nabla p}{p}\right) \frac{\nabla p}{p}\right) \\
& =\frac{\sigma^{2}}{2}\left(\Delta \frac{\nabla|\Psi|^{2}}{|\Psi|^{2}}+\left(\partial_{x} \frac{\nabla|\Psi|^{2}}{|\Psi|^{2}}\right) \frac{\nabla|\Psi|^{2}}{|\Psi|^{2}}\right) \\
& =\sigma^{2}\left(\Delta \frac{\nabla|\Psi|}{|\Psi|}+2\left(\partial_{x} \frac{\nabla|\Psi|}{|\Psi|}\right) \frac{\nabla|\Psi|}{|\Psi|}\right) \\
& =\sigma^{2} \nabla \frac{\Delta|\Psi|}{|\Psi|} .
\end{aligned}
$$

Since $b$ is a gradient, we can again use the formula

$$
\overline{\mathcal{D}}^{2} X_{t}=i \sigma^{2} \nabla\left(\frac{\partial_{t} \Psi}{\Psi}-i \frac{\sigma^{2}}{2} \frac{\Delta \Psi}{\Psi}\right)\left(t, X_{t}\right)
$$

which is real, that is

$$
\Im\left(i \sigma^{2} \nabla\left(\frac{\partial_{t} \Psi}{\Psi}-i \frac{\sigma^{2}}{2} \frac{\Delta \Psi}{\Psi}\right)\right)=0 .
$$

Therefore

$$
\mathcal{D}_{0}^{2} X_{t}=-\sigma^{2}\left(i \partial_{t} \frac{\nabla \Psi}{\Psi}+\frac{\sigma^{2}}{2} \nabla \frac{\Delta \Psi}{\Psi}-\sigma^{2} \nabla \frac{\Delta|\Psi|}{|\Psi|}\right) .
$$

Since $\mathcal{D}_{0}^{2} X_{t}=-\nabla U\left(X_{t}\right)$, we then obtain by a suitable integration the desired nonlinear Schrödinger equation. 


\section{Application : organisation of planetary systems}

In order to apply the stochastisation hypothesis, we must find physical systems for which something about the underlying set of stochastic processes can be said. Of course, using different operators we can enlarge the class of stochastic processes which can be considered. However, two examples related to the dynamics of planetary systems can be studied : the protoplanetary nebula dynamics and the long term behaviour of the solar system. The following Sections give details for these two cases.

5.1. Dynamics of a protoplanetary nebula. - We follow the work of S. Albeverio and al. [3] as well as J. Laskar [29] and L. Nottale [42]. In both of these models the main goal is to derive the general features of the organization of planetary systems without a precise mechanism for accretion. Our main problem in order to apply the stochastisation hypothesis is to characterize the underlying set of stochastic processes for the dynamics of a protoplanetary nebula. In the next Section, we discuss this problem following S. Albeverio and al. [3], J. Laskar [29] and L. Nottale [42].

5.1.1. Model for the dynamics in a protoplanetary nebula. - We first make our basic assumptions on the structure of the protoplanetary nebula:

Structural assumptions : A protoplanetary nebula is made of :

- A central body of mass $M$ acting by some potential $U$.

- A gas of some particles called grains around the central body.

We also make some assumptions on the dynamics of grains :

Dynamical assumptions : The motion of a given grain is governed by :

- The potential $U$.

- Collisions between grains.

The main point is of course to precise the nature of the collisions between grains. This is the subject of the next Section.

5.1.2. On the nature of collisions. - In the following, we make a simple assumption on the nature of collisions which enables us to use the results of Section 4. However, the same framework can be used under more general assumptions. 
Assumptions on collisions : We assume that the collisions are :

- Randomly distributed

- Isotropic

- Homogeneous

As a consequence of isotropy and homogeneity, we can model the randomness by a white noise (see [3], p. 366). Hence, the typical motion of a grain can be modelled by a diffusion process with a constant diffusion coefficient (due to isotropy and homogeneity). These assumptions then induce that we work with diffusion processes in $\Sigma_{\sigma}, \sigma \in \mathbb{R}$.

5.1.3. Mechanisms for confinements. - The previous Section allows us to use the reasoning made by S. Albeverio and al. [3] and used also by L. Nottale [41] in order to prove that the dynamics of a protoplanetary nebula naturally leads to formation of structures which follow a simple rule. We give a short reminder of the results described in details in Albeverio and al. ([4],§.2, p. 193-197) concerning the formation of impenetrable barriers.

We denote by

$$
B_{\psi}=\left\{x \in \mathbb{R}^{3}, \psi(x)=0\right\}
$$

Let $X_{t}$ be a process starting in $x \notin B_{\psi}$. Then under general assumptions on $U$, one can prove that $X_{t}$ never reaches $B_{\psi}$. We can then define disjoint connected regions $\Lambda_{n}$ such that

$$
\mathbb{R}^{3}=B_{\psi} \cup\left(\bigcup_{n} \Lambda_{n}\right) .
$$

The previous result on $B_{\psi}$ leads to the following confinement property :

Confinement property : If $X_{t}$ is a process starting in $x \in \Lambda_{n}$, then $X_{t} \in \Lambda_{n}$ for all $t$.

This mechanism is already used in quantum mechanics.

Remark 9. - In the paper of J. Laskar [29] the organisation of the system is related to the conservation of the angular momentum deficit ( $A M D$ ) for the averaged equations, the main remark being that the erratic wandering of orbits are still constrained by the conservation of energy and angular momentum. As noticed by J. Laskar ([28],p.I.11 and [29], p.3243) this provides a kind of confinement property using the fact that the motion of the large planets is very regular. Indeed, a large excursion of the inner planets would induce a big variation of the angular momentum for the outer planets, which is more or less constant. 
Remark 10. - As proved in Section 2.5.5, the stochastic embedding preserved the invariance of the Lagrangian under rotations and as a consequence, using the stochastic Noether's theorem, the components of the angular momentum are also first integrals for the stochastic Lagrangian system. The previous reasoning of J. Laskar can then be applied in our stochastic setting.

5.1.4. Law of repartition for planetary orbits. - We do not reproduce here the computations and remarks which lead the law of repartition for planetary orbits using the Schrödinger equation. These arguments can be found in L. Nottale [42] and S. Albeverio and al. [4]. The distribution of the distances of planets takes the form

$$
\sqrt{a}=n\left(1+\frac{1}{2 n}\right)^{1 / 2} \sqrt{a_{0}},
$$

or

$$
\sqrt{a}=n \frac{2 \sigma}{\sqrt{g M}},
$$

where

$$
\sqrt{a_{0}}=\frac{4 \sigma^{2}}{g M},
$$

depending on the fact that one considers the mean distance or the peak of probability density.

For homogeneity reasons the constant $\sigma$ must have the dimension of a mass. However, the expected form of this constant is not given in our setting. L. Nottale ([43], p.1021), using arguments related to the principle of equivalence, propose to look for this constant in the following form :

$$
\sigma=\frac{g M}{2 \omega_{0}},
$$

where $\omega_{0}$ has the dimension of a velocity.

5.2. Long term behaviour of the solar system. - The main problem here is to give some prediction about the long term behaviour of the solar system. As noticed by J. Laskar ([28], p. L9) as the motion of the solar system is chaotic with a Lyapounov time of about $5 \mathrm{Myr}$ a numerical integration must be only "considered as an indication of its possible behaviour, and cannot pretend to be the description of its actual motion.". As a consequence, one must perform many numerical integrations in order to obtain a global view of the dynamics of the solar system. In this Section, using the numerical results of J. Laskar on the chaotic behaviour of the solar system, we bypass this problem by including the randomness induced by chaos using the stochastisation hypothesis. Doing so, we must divide the solar system in two parts : the large planets and the inner planets due to their different dynamical behaviour. We then 
obtain again a law of repartition for the semimajor axes of the planets of the solar system which follow a $n^{2}$ power law for the outer and inner planetary system.

5.2.1. Chaos and Stochastic processes. - The long term behaviour of the solar system is chaotic. Following J. Laskar ([29]) it has the following consequence: "Since the characteristic time scale for the divergence of nearby orbits in the Solar system is approximately $5 \mathrm{Myr}$, the orbital evolution of the planets becomes practically unpredictable after 100 Myr. Thus in the long term, the motion of the solar system may be described by a random process, where orbits wander erratically in a chaotic zone.". It is well known that in some cases, the long term dynamics can be associated to diffusion processes like for example in the Lorenz gas [61] or some problems of instability of Hamiltonian systems [49]. In the following, we make the assumption that the set of stochastic processes is a subset of diffusion processes.

For more general assumptions, we refer to the work of G. Zaslavsky [61] on Hamiltonian Chaos and Fractional dynamics which describes the possible characteristics of these stochastic processes. For a work in the spirit of the stochastisation hypothesis about Hamiltonian Chaos, we refer to [15].

Remark 11. - In (443], Section 2., p.1019) L. Nottale describes an heuristic method to associate stochastic processes to a chaotic dynamical system. These heuristic arguments lead to diffusion processes. Indeed, the idea is to look for trajectories of the dynamical system over a time which is far larger than the Lyapounov time. As a consequence, the resulting trajectory "become" Markovian. General assumptions on the nature of the "fluctuation" term lead then to diffusion processes.

5.2.2. Hierarchical structures. - The main consequences of the numerical simulations made by J. Laskar (see for example [28]) is a fundamental difference between the dynamical behaviour of the large planets and the inner planets (see [28], Fig. 1a, p.I.10). The motion of the large planets is always (up to $200 \mathrm{Myr}$ ) very regular. On the other hand, the motion of the inner planets is highly chaotic. As a consequence, the stochastisation hypothesis can be applied using different diffusion coefficients $\sigma_{\text {in }}$ and $\sigma_{\text {out }}$ leading to two $n^{2}$ power law for the law of repartition for the semimajor axes of planets.

As remind by J. Laskar ([29], p. 3243), "it is well known that, if the Solar system is split into sets of inner and outer planets, the seminmajor axes in each set follow a $n^{2}$ power law to a high degree of approximation" (see also [46],[50]). However, the previous splitting is not assumed here, but follows from the stochastisation hypothesis as the underlying set of stochastic processes have not the same diffusion coefficient. 
Remark 12. - The theory developed by L. Nottale predicts a simple relation between these two coefficients :

$$
\sigma_{\mathrm{out}}=5 \sigma_{\mathrm{in}}
$$

This result can not follow from our formalism or the one used by J. Laskar and S. Albeverio and al.

5.2.3. Is the Solar system stable ? - Following the formulation of Moser [36], the problem of the stability of the solar system is "the question of deciding whether the planetary system in the distant future will keep the same form as it now has or whether after a long time perhaps one or another of the planets might leave the solar system or whether collisions might even lead to a catastrophic change."

From a mathematical point of view, the problem remains open. The Arnold's theorem on the stability of $n$-body problem [1] does not give a significant result as it applies under unrealistic assumptions [24]. We refer to [19] for a complete proof of Arnold's theorem following Herman's approach and in particular Section 8, p.61-62 for comments about the stability of the solar system.

However, as pointed out by D. Mumford in ([37], §.6) the classical study of the $n$-body problem is not sufficient in order to have a more realistic model for the dynamics of planetary motion. He says that Newton's laws of motion "... predicted wonderfully planetary motion and, with perturbation, models the full set of planets for moderate period of time (e.g., maybe $10^{8}$ years). But going out further (maybe to $10^{9}$ years), the unmodeled effects begin to add up and the approximation is not useful. So where does this leave the mathematical study of the 3-body problem ? It makes the classical deterministic analysis of the 3-body gravitational equations about as relevant to the world as the continuum hypothesis! A major step in making the equation more relevant is to add a small stochastic term.".A study of a stochastically perturbed two-body problem is done in [57].

Remark 13. - To add a small stochastic term is not trivial and the significance of this adding is far from being well understood. In particular, the word "perturbation" seems to be an abuse of language because the relation between the perturbed equation and the initial deterministic equation is not so easy and far from intuition (I refer to the classical phenomenon described by Wong and Zakai [59]) and also because the perturbed object and the initial one do not belong to the same class of objects. A main problem also is in which sense we must consider the perturbation (Itô or Stratonovich or something else intermediary). This problem is fundamental in many applied problems, in particular in biology. It must be noted that in 
the stochastic embedding framework, the classical equation and then the classical motion is contained in the stochastic version.

From the numerical point of view Laskar [30] has proved that collisions can occur between Mercury, Mars, Venus and the Earth. A review of several results of Laskar and others can be found in Marmi [33] and Laskar [31]. The remark of Mumford applies again as no stochastic perturbations of the equations have been taken into account for the numerical simulation of the planetary motion. However, several other physical effects, in particular relativistic effects, are integrated. In general, due to the Chaotic evolution of the solar system, we can only, taking a set of initial conditions, provide some possible evolutions of the behaviour of the planets. The probability of, for example, collisions between the Earth and Venus or Mercury is not known. We can only say that such a collision is possible.

Our result provides a stability result in a probabilistic sense : regarding the solar system over stochastic processes we loose a precise location of the planets. However, we obtain in expectation the fact that the structure of this system remains the same. This does not prevent the system to experience catastrophic behaviour as found by Laskar. Of course, our result is only a first step in a complete stochastic approach to the stability problem of the solar system. We have to assume that the dynamical effect induced by the others planets, i.e. mutual gravitational interaction, on the dynamical motion of a single planet can be modeled by a particular class of stochastic processes at least over a sufficient large amount of time. A more realistic approach is certainly to consider the complete $n$-body problem, to make a stochastic embedding and then a stochastic perturbation. We discuss in more details this perspective in Section 6.2.3.

\section{Conclusion}

6.1. Summary. - The previous approach to the organisation of planetary systems based on the stochastisation hypothesis and the formalism of stochastic embedding provides a unified treatment of several approaches, in particular the work of S. Albeverio and al. [3] and L. Nottale [42]. As discussed in this paper, it allows us also to either simplify or reduce the number of assumptions or to give complete proofs for partially justified results. We can list the following points :

- The form of the stochastic equation is fixed by embedding and preserves the form as well as the variational structure of the initial equation. This last result is not assumed but proved. 
- The form of the stochastic acceleration is fixed by the theory and not assumed as in S. Albeverio and al. [3].

- The density probability of the stochastic processes which are solutions of the stochastic Newton's equation is proved to satisfy the Schrödinger equation.

- The same formalism can be applied to the long term behaviour of the Solar system providing a stability result for planetary orbits contrary to all others known approaches.

- Finally, only one assumption is used : the stochastisation hypothesis which is heuristically used in many arguments but mathematically formalized in our framework using the stochastic embedding formalism.

\subsection{Perspectives. -}

6.2.1. Coalescent processes. - This framework can be developed in several directions. The main one is certainly to study the stochastic Newton's equation over more general stochastic processes than diffusion processes. In particular, with respect to the simple assumption made in Section 5.1.2 and related to the accretion mechanisms, we can for example assume as in J. Laskar ([29], p.3240) that when two small bodies of mass $m_{1}$ and $m_{2}$ collide, they form a new one of mass $m_{1}+m_{2}$. A natural choice for the underlying stochastic processes is then given by coalescent processes (see for example [6]).

6.2.2. Statistics and gravitational structuration constant. - Another important point is to compute the constant of structure $\omega_{0}$ in the law (50) and the integers $n$ for every Planet of extrasolar planetary systems and to perform a statistical analysis in details. The main point is to give a definitive confirmation for the existence of a universal constant governing the organisation of gravitational structures predict by L. Nottale $([\mathbf{4 2}],[\mathbf{4 4}])$. This result is not predicted or discussed in the other approaches to the formation of planetary systems. However, the statistical analysis of this problem is not simple and leads to many difficulties. For an example of the typical difficulties related to this problem, we refer to B. Efron [18], I.J. Good [22] and W. Hayes-S. Tremaine [23].

6.2.3. Stability of the solar system. - As already discussed, the previous result on the existence of a law of repartition of planetary orbits gives a hint that the solar system is stable in a probabilistic sense. Due to the Chaotic behaviour of the solar system, we can not hope more that this result. Of course, as pointed out, many assumptions are not mathematically proved, in particular the existence and properties of the stochastic processes associated to the chaotic behaviour of the solar system are far from being characterized. In this paper, we have only made assumptions which are satisfied by chaotic Hamiltonian systems under general 
hypothesis. However, even if we manage to describe such class of processes, this result is by itself not sufficient. Indeed, we must also make a perturbation of the stochastic system which is then described, as the underlying model is not complete. As a consequence, the problem is to develop the stochastic theory of perturbations for stochastic dynamical systems obtained by stochastic embedding.

\section{References}

[1] V.I. Arnold. Petits dénominateurs et problèmes de stabilité du mouvement en mécanique classique et céleste (en russe). Usp. Mat. Nauk. 18 (1963), 91Ű192 (trad. anglaise, Russ. Math. Surv. 18 (1963), 85Ü193).

[2] Arnold V.I., Mathematical Methods of Classical Mechanics, 2d edition, Springer, 1989.

[3] Albeverio S., Blanchard Ph., Hoegh-Krohn R., A stochastic model for the orbits of planets and satellites: an interpretation of Titius-Bode law, Expositiones Mathematicae 4, 363-373 (1983).

[4] Albeverio S., Blanchard Ph., Hoegh-Krohn R., Newtonian diffusions and planets, with a remark on non-standard Dirichlet forms and polymers,

[5] Arnold, Ludwig . Random dynamical systems. Springer Monographs in Mathematics. SpringerVerlag, Berlin, 1998. 586 pp.

[6] Bertoin J. J-F. Le Gall, Stochastic flows associated to coalescent processes, Probab. Th. Rel. Fields 126 (2003), 261-288.

[7] Eric A. Carlen. (1984) Conservative diffusions. Comm. Math. Phys. 94, no 3, 293-315.

[8] J. Cresson, Non-differentiable deformations of $R^{n}$, International Journal of Geometric Methods in Modern Physics, Vol. 3, no. 7 (2006) 1395-1415.

[9] J. Cresson and S. Darses (2006), Plongement stochastique des systèmes lagrangiens, C.R. Acad. Sci. Paris Ser. I 342 (5), 333-336.

[10] J. Cresson and S. Darses, Stochastic embedding of dynamical systems. J. Math. Phys., 48(7):072703, 54, 2007.

[11] J. Cresson, S. Darses, Théorème de Noether Stochastique, C.R. Acad. Sci. Paris, Ser. I 344 (2007) 259-264

[12] J. Cresson, Introduction to embedding of Lagrangian systems, International Journal of Biomathematics and Biostatistics, Vol. 1, no. 1, 23-31, 2010

[13] J. Cresson, Introduction to embedding formalisms and Lagrangian PDEs, Lecture Notes TU München, 2011.

[14] J. Cresson, I. Greff, A non-differentiable Noether's theorem, J. Math. Phys. 52, 023513 (2011); doi:10.1063/1.3552936 (10 pages).

[15] J. Cresson and P. Inizan. About fractional Hamiltonian systems. Physica Scripta, T136:014007, 2009.

[16] S. Darses and I. Nourdin (2006): Stochastic derivatives for fractional diffusions. To appear in Ann. Probab.

[17] S. Darses and I. Nourdin, Dynamical properties and characterization of gradient drift diffusions. Electron. Comm. Probab. 12 (2007) 390-400

[18] Efron B., Does an observed sequence of numbers follow a simple rule ? (another look at Bode's law), J. Am. Statist. Assoc. 66 (335), 552-559, 1971.

[19] Féjoz J., Démonstration du "théorème d'Arnold" sur la stabilité du système planétaire (d'après M.Herman), Ergod. Th. and Dynam. Sys. (2004), 24, 1-62. 
[20] H. Föllmer (1984): Time reversal on Wiener space. Stochastic processes - mathematics and physics (Bielefeld). Lecture Notes in Math. 1158, 119-129.

[21] E. Fournié, J.-M. Lasry, J. Lebuchoux, P.-L. Lions and N. Touzi (1999): Applications of Malliavin calculus to Monte Carlo methods in finance. Finance Stochast. 3, 391-412.

[22] Good I.J., A subjective evaluation of Bode's law and an "objective" test for approximate numerical rationality, J. Am. Statis. Assoc. 64, 23-66, 1969.

[23] W. Hayes, S. Tremaine, Fitting selected random planetary systems to Titius-Bode laws, Icarus 135, 549-557 (1998).

[24] M. Hénon. Exploration numérique du problème restreint IV. Masses égales, orbites non périodiques. Bull. Astronom. 3(1 :2) (1966), 49-66.

[25] I. Karatzas and S.E. Shreve (1991): Brownian Motion and Stochastic Calculus. Springer-Verlag, New York Second Edition.

[26] K. Itô, On stochastic differential equations, Mem. Amer. Math. Soc. 4 (1951), 1-51.

[27] A.N. Kolmogorov (1937): Zur Umkehrbarkeit der statistischen Naturgesetze. Math. Ann 113, 766-772.

[28] Laskar J., Large-scale chaos in the solar system, Astron. Astrophys. 287, L9-L12 (1994).

[29] Laskar J., On the spacing of planetary systems, Phys. Rev. Lett. 84? no. 15, 3240-3243, 2000.

[30] J. Laskar, M. Gastineau, Existence of collisional trajectories of Mercury, Mars and Venus with the Earth, Nature Vol. 459, 2009.

[31] Jacques Laskar, Le Système solaire est-il stable ?, Séminaire Poincaré XIV (2010) 221-246.

[32] Levy-Leblond J-M, One more derivation of the Lorentz transformation, Am. J. Phys. Vol. 44, No. 3, 1976, pp. 271-277.

[33] Marmi, S., Chaotic behaviour in the solar system following J. Laskar, Séminaire Bourbaki, 51ème année, 1998-1999, Paper No. 854.

[34] A. Millet, D. Nualart and M. Sanz (1989): Integration by parts and time reversal for diffusion processes. Ann. Probab. 17, no. 1, 208-238.

[35] Misawa T., Yasue K., Canonical dynamical systems. J. Math. Phys. 28(11), 1987, 2569-2573.

[36] Moser, J., Is the solar system stable?, Math. Intell. 1, 65Û́71, 1978.

[37] Mumford D., The dawning of the age of stochasticity, in Mathematics: Frontiers and perspectives, V. Arnold, M. Atiyah, P. Lax, B. Mazur editors, AMS, 2000, 197-218.

[38] E. Nelson (2001): Dynamical theory of Brownian motion. Princeton University Press. Second edition. Available online at http://www.math.princeton.edu/ nelson/books/bmotion.pdf.

[39] Nelson E., Derivation of the Schrödinger equation from Newtonian mechanics, Physical Review, Vol. 150, No. 4, 1079-1084 (1966).

[40] Nottale L., Fractal Space-Time and Microphysics : towards a theory of Scale relativity, World Scientific, 1993.

[41] Nottale L, New formulation of stochastic mechanics. Application to chaos, in "Chaos and diffusion in Hamiltonian systems", Proceedings of the fourth workshop in Astronomy and Astrophysics of Chamonix (France), 7-12 February 1994, Eds. D. Benest and C. Froeschlé (Editions Frontières), pp. 173-198 (1995).

[42] Nottale L., The quantization of the solar system, Astron. Astrophys. 315, L9, 1996.

[43] Nottale L., Scale relativity and quantization of the solar system, Astronomy and Astrophysics. 322, 1018-1025 (1997).

[44] Nottale L., Scale-relativity, Fractal space-time and gravitational structures, in "Fractals and Beyond : complexity in the sciences", Ed. M.M. Novak, pp. 149-160, World Scientific, 1998.

[45] Nottale L., La relativité dans tous ses états, Hachette littérature, 2000.

[46] J.C. Pecker, E. Schatzman, Astrophysique générale, Masson, Paris, 1959. 
[47] I. Prigogine, Les lois du chaos, Champs Flammarion, 1994.

[48] S. Roelly and M. Thieullen (2005): Duality formula for the bridges of a Brownian diffusion: application to gradient drifts. Stochastic Process. Appl. 115, no. 10, 1677-1700.

[49] Sauzin D., Exemples de diffusion d'Arnold avec convergence vers un mouvement brownien, Journées scientifiques 2006 de l'Institut de Mécanique Céleste et de Calcul des éphémérides, Notes scientifiques et techniques de l'Institut de Mécanique Céleste.

[50] O. Schmidt, in The origin of the Solar system : Soviet Research, 1925-1991, Edited by A.E. Levin and al., AIP, New York, 1995.

[51] Stroock, Daniel W. ; Varadhan, S. R. Srinivasa . Multidimensional diffusion processes. Reprint of the 1997 edition. Classics in Mathematics. Springer-Verlag, Berlin, 2006. xii +338 pp.

[52] Thieullen, M. and Zambrini, J. C. (1997): Symmetries in the stochastic calculus of variations. Probab. Theory and Rel. Fields 107, no 3, 401-427.

[53] Thieullen M. and Zambrini J.C, Probability and quantum symmetries I. The theorem of Noether in Schrödinger's euclidean mechanics, Ann. Inst. Henri Poincaré, Physique Théorique, Vol. 67, 3, p. 297-338 (1997).

[54] Wu, Liming. Uniqueness of Nelson's diffusions (1999). Probab. Theory Related Fields, 114, no. $4,549-585$.

[55] L.C.G. Rogers and D. Williams (1987). Diffusions, Markov processes, and martingales. Vol. 2. Itô calculus. Wiley Series in Probability and Mathematical Statistics: Probability and Mathematical Statistics. John Wiley and Sons, Inc., New York. 475 pp.

[56] L. Schwartz, Théorie des distributions, Publications de l'Institut de Mathématique de l'Université de Strasbourg, No. IX-X. Nouvelle édition, entiérement corrigée, refondue et augmentée, Hermann, Paris, 1966.

[57] N Sharma and H Parthasarathy, Dynamics of a stochastically perturbed two-body problem, Proc. R. Soc. A 2007 463, 979-1003.

[58] J-C. Zambrini. Variational processes and stochastic versions of mechanics. J. Math. Phys. 27 (1986), no. 9, 2307-2330.

[59] Wong E., Zakaï M., On the relationship between ordinary and stochastic differential equations, Internat. J. Engin. Sci. 3, 213-229, 1965.

[60] K. Yassue, Stochastic calculus of variations, Journal of functional analysis 41, 327-340 (1981).

[61] G.M. Zaslavsky. Hamiltonian Chaos 83 Fractional Dynamics. Oxford University Press, Oxford, 2005.

[62] Zheng W.A., Meyer P.A., Quelques résultats de mécanique stochastique. Séminaire de Probabilités XVIII, 223-243

JaCky Cresson ${ }^{1,2},{ }^{1}$ Laboratoire de Mathématiques Appliquées de Pau, Université de Pau et des Pays de l'Adour, avenue de l'Université, BP 1155, 64013 Pau Cedex, France • ${ }^{2}$ Institut de Mécanique Céleste et de Calcul des Éphémérides, Observatoire de Paris, 77 avenue Denfert-Rochereau, 75014 Paris, France 